\title{
Maffucci syndrome: Anaesthetic management and review of literature
}

\author{
Gyaninder P. Singh, Barkha Bindu, Mihir P. Pandia, Parmod K. Bithal
}

\begin{abstract}
Maffucci syndrome is a rare, nonhereditary disorder manifesting early in life.The syndrome is characterized by presence of multiple hemangiomas and enchondromas mostly affecting the extremities. Haemangiomas are usually cutaneous, but may sometimes be visceral as well. Enchondromas commonly affect the long bones of the extremities. However, other areas including skull, ribs, vertebrae, larynx and trachea may also be involved. The presence of these lesions in the trachea and/or oropharynx may compromise the airway and cause difficulty during tracheal intubation. Complete airway examination and investigation is important to rule out any such lesion in a patient of Maffucci syndrome before planning for general anaesthesia with tracheal intubation.
\end{abstract}

Key words: Enchondromas, haemangiomas, Maffucci syndrome, tracheal intubation

\section{INTRODUCTION}

Maffucci syndrome is a rare, sporadic, nonhereditary disease, primarily affecting bones and skin. Since its first description in 1881, approximately 200 cases have been reported in the medical literature worldwide. It is characterized by multiple enchondromas associated with multiple haemangiomas and sometimes, lymphangiomas as well. Enchondromas most commonly occur in the limb bones, especially in the hands and feet and pathological fractures are common in the affected long bones. However, they may also occur in skull, ribs and vertebrae. Affected individuals may develop bone cancers called chondrosarcomas, which is the most frequently encountered malignancy in Maffucci syndrome. ${ }^{[1]}$ They also have an increased risk of other cancers, such as ovarian or liver cancer. We report here a patient of Maffucci syndrome who apart

\begin{tabular}{|l|l|}
\hline \multicolumn{2}{|c|}{ Access this article online } \\
\hline Quick Response Code: & Website: \\
\hline & www.jnaccjournal.org \\
\cline { 2 - 2 } & \\
\hline
\end{tabular}

from the bone and skin abnormalities, had tracheal findings as well.

\section{CASE REPORT}

A 26-year-old female, weighing $79 \mathrm{~kg}$, who had been diagnosed with Maffucci syndrome 10 years ago, presented to us with complaints of gradual reducing vision in right eye since 2 years without field defect. At the time of presentation, she only had perception of light in right eye. The patient was undergoing treatment for multiple haemangiomas with laser ablation for the past 10 years and had been operated for granulosa cell tumour of ovary at the age of 25 years. She had also undergone amputation of little finger of the left hand for painful enchondroma, 1-month before presenting to us. On evaluation for reduced vision, she was found to have sellar intraventricular chondrosarcoma and was planned for craniotomy and excision of tumour.

The patient was obese with short neck, double chin and Mallampati grade IV. Because of the anticipated difficult intubation and a possibility of accidental trauma to the undetected intraoral haemangiomas, fibreoptic bronchosopy and intubation were planned. The patient was premedicated with glycopyrrolate $(0.2 \mathrm{mg}$ intramuscular) and ranitidine (50 $\mathrm{mg}$ intravenous), half an hour

Department of Neuroanaesthesiology, All India Institute of Medical Sciences, New Delhi, India

Address for correspondence:

Dr. Mihir Prakash Pandia, Department of Neuroanaesthesiology, Room No. 709 ( $7^{\text {th }}$ Floor), Neurosciences Centre, All India Institute of Medical Sciences, New Delhi - 110 029, India. E-mail: pandiamihir@gmail.com 
before the start of surgery. In the operating room, routine monitors were attached and the patient was induced with fentanyl $180 \mathrm{mcg}$ and propofol $150 \mathrm{mg}$. Rocuronium (1 mg/kg) was administered to facilitate bronchoscopy and tracheal intubation. During fibreoptic bronchoscopy two small nodular growths of about $5 \mathrm{~mm} \times 5 \mathrm{~mm}$ were noticed in the tracheal wall at mid trachea level [Figure 1a]. Another longitudinal projection extending distally along the tracheal wall into the lumen was also seen [Figure 1b]. However, these findings did not impede intubation, and a $7.0 \mathrm{~mm}$ internal diameter cuffed portex endotracheal tube was railroaded over the fibreoptic bronchoscope without resistance. Anaesthesia was maintained using sevoflurane (0.8-1.2 minimum alveolar concentration) in a mixture of oxygen and nitrous oxide (1:2) along with intermittent boluses of fentanyl and vecuronium. Intraoperative course of the surgery remained uneventful except, for multiple transient episodes of bradycardia (caused by stimulation of the hypothalamus during resection of the tumour in this region). However, the blood pressure remained within the normal range throughout. Intraoperative findings of the surgery revealed a bone tumour of variable consistency in suprasellar space involving right optic nerve, internal carotid artery and right cavernous sinus, extending into prepontine space. The surgery lasted for $9 \mathrm{~h}$. Total blood loss during the surgery was about $500 \mathrm{ml}$ which was adequately replaced with fluids and one unit of packed red blood cells. At the end of the surgery, decision to mechanically ventilate the patient was made because of residual tumour mass and handling of hypothalamus during the surgery and the patient was shifted to Intensive Care Unit for elective mechanical ventilation and monitoring.

\section{DISCUSSION}

Ollier first described cases of multiple enchondromatosis, later referred to as Ollier disease. When multiple enchondromas are accompanied by multiple haemangiomas, the condition is called as Maffucci syndrome (first described by Angelo Maria Maffucci in 1881).

Maffucci syndrome is a rare congenital entity usually manifesting in early childhood. It is nonfamilial disease occurring spontaneously. The definitive etiology is still unknown. A mutation in the Indian hedgehog and parathyroid hormone-related protein pathway is thought to be the causative factor. ${ }^{[1]}$

The haemangiomas in Maffucci syndrome are usually cutaneous but can sometimes be visceral as well. They frequently protrude as soft bluish nodules over the distal extremities [Figure 1c] but can appear anywhere in the body. They may sometimes be present in the

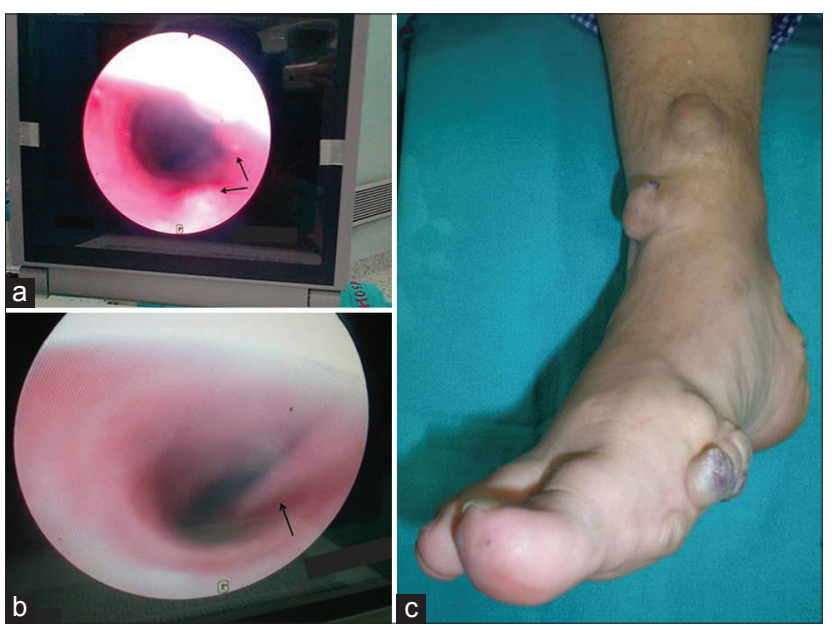

Figure 1: (a) Arrows showing the nodular swellings projecting into the tracheal lumen. (b) Arrow showing a longitudinal crest projecting into the lumen of trachea from the tracheal wall. (c) Multiple cutaneous hemangioma over the foot

oropharynx and trachea also. Enchondromas form when previously normal cartilage in the epiphyseal growth plates or suture lines of the skull fails to undergo normal ossification or undergoes malignant degeneration, and can result in deformities such as short stature, scoliosis, and pathologic fractures. ${ }^{[1]}$ The most common intracranial manifestation of enchondromas is in the sphenoid bone. Enchondromas may also arise from the sinonasal tract, petrous apex, cerebellopontine angle and mandible..$^{[2]}$ Common presenting symptoms of intracranial enchondromas are headache, dizziness, tinnitus, and hearing loss. Cranial nerve involvement causing visual deficits may also occur. ${ }^{[3]}$ Malignant transformation to chondrosarcoma must be suspected when cartilaginous lesions show continued growth after normal skeletal growth has stopped or when patient complains of pain. ${ }^{[2]}$ Enchondromas and chondrosarcomas are less frequent than vascular malformations. Cartilaginous tumours associated with Maffucci syndrome may arise in the larynx and trachea. ${ }^{[4]}$ Maffucci syndrome is also associated with a high risk of development of central nervous system, pancreatic, and ovarian malignancies.

The treatment of choice for intracranial chondrosarcomas is surgical excision, but the chances of postoperative morbidity are very high due to the close proximity of the lesions to critical intracranial structures including the brainstem, cranial nerves and great vessels of the neck. Thus, debulking of the tumour is the primary goal of treatment, thereby, minimizing the chances of iatrogenic injury to critical neurovascular structures. Remnant tumour might require adjuvant radiotherapy later. ${ }^{[5]}$

The important anesthetic concerns in Maffucci syndrome have been implicated to the presence of haemangiomas 
and enchondromas. It is important to check for the presence of haemangiomas in the upper airway during preanesthetic checkup. Fibreoptic bronchoscopy and intubation must be preferred in patients with haemangiomas in the oral cavity and contact of the bronchoscope or the tube with hemangiomas must be prevented to avoid rupturing of any haemangiomas. Careful positioning is required to avoid pathological fractures.

Tracheal chondromas and chondrosarcomas arise from tracheal cartilaginous rings and may cause obstructive symptoms depending on whether the tumour grows intra-luminal or extends through the tracheal wall. ${ }^{[6]}$ Chondrosarcomas of the trachea are slow growing and do not metastasize, in contrast to chondrosarcomas found elsewhere in the body. ${ }^{[6]}$ Obstructive lesions of the trachea can cause stridor and wheeze when $>75 \%$ of the tracheal lumen is compromised. Computed tomography scan is a good modality to visualize these lesions and provides accurate information about the location and extension of the lesions. ${ }^{[6]}$ If a cartilaginous tumour is discovered in the larynx or trachea, frequent debulking of cartilaginous mass might be required to maintain patency of the airway. However, complications like tracheomalacia and tracheal stenosis might occur. ${ }^{[4]}$

We report this case to highlight the fact that unexpected difficult intubation may be encountered in these patients. In our patient, we came across small nodular growths and a longitudinal crest bulging into the tracheal lumen from the tracheal wall while performing the fibreoptic intubation. Such growths, if large in size may compromise the tracheal lumen and cause unexpected complications like failed intubation or tracheal bleeding due to trauma. We would like to emphasize that apart from the presence of cutaneous haemangiomas and enchondromas over the extremities and intracranial region, presence of oral, laryngeal and/or tracheal growths must also be considered and ruled out preoperatively even in the absence of obstructive symptoms. Thus, thorough airway examination, proper planning and preparation before taking the patients of Maffucci syndrome for anaesthesia cannot be over emphasized.

\section{REFERENCES}

1. Garzon MC, Huang JT, Enjolras O, Frieden IJ. Vascular malformations. Part II: Associated syndromes. J Am Acad Dermatol 2007;56:541-64.

2. Sun GH, Myer $\mathrm{CM} 3^{\text {rd }}$. Otolaryngologic manifestations of Maffucci's syndrome. Int J Pediatr Otorhinolaryngol 2009;73:1015-8.

3. Abdelmalek M, Stanko C. Recurrent chondrosarcoma of the right skull base in a patient with Maffucci syndrome. Am J Clin Dermatol 2008;9:61-5.

4. Moore BA, Rutter MJ, Cotton R, Werkhaven J. Maffucci's syndrome and cartilaginous neoplasms of the trachea. Otolaryngol Head Neck Surg 2003;128:583-6.

5. Dini LI, Isolan GR, Saraiva GA, Dini SA, Gallo P. Maffucci's syndrome complicated by intracranial chondrosarcoma: Two new illustrative cases. Arq Neuropsiquiatr 2007;65:816-21.

6. Wagnetz U, Patsios D, Darling G, Las Heras F, Hwang D. Tracheal chondrosarcoma - A rare complication in Maffucci syndrome. Br J Radiol 2009;82:e178-81.

How to cite this article: Singh GP, Bindu B, Pandia MP, Bithal PK. Maffucci syndrome: Anaesthetic management and review of literature. J Neuroanaesthesiol Crit Care 2015;2:136-8.

Source of Support: Nil, Conflict of Interest: None declared. 